\title{
La Realidad Aumentada: tecnología emergente para la sociedad del aprendizaje
}

\section{The Augmented Reality: Emerging Technology for the Learning Society}

\author{
Julio Cabero Almenara cabero@us.es — http://orcid.org/0000-0002-1133-603 \\ Universidad de Sevilla, España \\ Angel Puentes Puente ap6348@unphu.edu.do — https://orcid.org/0000-0001-7575-5625 \\ Universidad Nacional Pedro Henríquez Ureña, República Dominicana
}

Fecha de recepción: 4 de abril de 202

Fecha de aceptación: 28 de abril de 2020

Fecha de publicación: 10 de julio de 2020

Favor de citar este artículo de la siguiente forma:

Cabero Almenara, J., y Puentes Puente, A. (2020).

La Realidad Aumentada: tecnología emergente para la sociedad del aprendizaje.

AULA, Revista de Humanidades y Ciencias Sociales, 66 (2), 35-51

\section{RESUMEN}

La adopción de tecnologías emergentes y la creación de modelos físicos mediante las tecnologías de realidad aumentada, constituyen en la actualidad una alternativa para los procesos de enseñanza aprendizaje. En este trabajo se presentan las características distintivas de la Realidad Aumentada (RA), su percepción de uso, así como los diferentes escenarios y niveles educativos en los cuales su uso nos brinda experiencias interesantes. Se expone el Proyecto RAFODIUN, proyecto de investigación de I+D+I financiada en el marco del Plan Estatal de Fomento de la Investigación Científica y Técnica de Excelencia 2013-2016, con referencia EDU2014-57446-P de la Universidad de Sevilla, donde se brindan las diferentes fases y resultados del estudio, así como las posibilidades que, desde el punto de vista de la investigación, se abren con su uso.

Palabras clave: Realidad Aumentada, recursos didácticos, objetos de aprendizaje, tecnologías emergentes, comunidad virtual, formación del profesorado.

\begin{abstract}
The adoption of emerging technologies and the creation of physical models using augmented reality technologies is currently an alternative to teaching-learning processes. This work presents the distinctive characteristics of AR, its perception of use, as well as the different educational settings and levels in which its use provides us with interesting experiences. The RAFODIUN Project is exposed, a R $+\mathrm{D}+\mathrm{I}$ research project funded within the framework of the State Plan for the Promotion of Scientific and Technical Research of Excellence 2013-2016, with reference EDU2014-57446-P of the University of Seville, where the different phases and results of the study are provided, as well as the possibilities that, from the point of view of the investigation, are opened with its use.
\end{abstract}

Keywords: Augmented Reality, teaching resources, learning objects, emerging technologies, virtual community, teacher training. 


\section{El auge significativo}

de la Realidad

\section{Aumentada (RA)}

En los comienzos del siglo XXI se están produciendo fuertes cambios en diferentes sectores, que van desde el trabajo profesional y las profesiones asociadas a él, en sectores como el Derecho, la Salud, o la Arquitectura (Suuskind y Susskind, 2016); la gestión y organización del conocimiento (Cabero, 2016); la educación con un nuevo imaginario social sobre la formación a distancia, la contemplación de nuevos escenarios de formación no limitándonos exclusivamente a los formales y la potenciación de un aprendizaje más profundo e híbrido; «la aparición de nuevas teorías de aprendizaje para explicar la adquisición del conocimiento en esta sociedad del aprendizaje, con la sustentación en el conectivismo, el aprendizaje ubicuo, el aprendizaje móvil, el aprendizaje rizomático o el aprendizaje invisible» $(\mathrm{Ca}-$ bero, Barroso y Llorente, 2016); hasta, cómo negarlo; la configuración de nuevos escenarios tecnológicos producto de la digitalización, el crecimiento exponencial de las tecnologías, la aparición del fenómeno de la web 2.0 y la significación que las TIC tienen en la sociedad del conocimiento.

En esta galaxia tecnológica en la cual nos nos hallamos, y que nunca nos habíamos encontrado en la historia de la humanidad, están emergiendo un bloque de tecnologías que van desde la web semántica, la internet de las cosas, y las analíticas de aprendizaje, hasta la RA. En el caso de esta última, podemos comprobar su significación por la importancia que se le concede en diferentes informes, como los «Horizonte» o el del Observatorio del Tecnológico de Monterrey sobre las tecnologías, donde señalan que impactará fuertemente a corto y mediano plazo en el sistema educativo (Tecnológico de Monterrey, 2015; Johnson y Adams, 2016; Johnson, Adams, Cummins, Estrada, Freeman, y Hall, 2016); o como en los hiperciclos de aceptación tecnológica que elabora anualmente la compañía Gartner. Allí también se puede reconocer que la RA ya ha superado el pico de expectativas sobredimensionadas y el abismo de desilusión y comienza a adentrarse en la rampa de la consolidación.

En definitiva, es posible aseverar que nos encontramos con una tecnología que está acercándose fuertemente a los escenarios de formación, sean estos presenciales o a distancia, e independientemente del nivel educativo donde se desarrolle la acción formativa y la disciplina que se imparta.

\section{La Realidad Aumentada \\ y sus características \\ distintivas}

Lógicamente hablar de un concepto, objeto o fenómeno, requiere comenzar por su especificación. Respecto a la RA, a la hora de su concreción lo primero es no caer en el error de creer que es de naturaleza reciente, pues ya en los años 90 el término se utilizaba en diferentes campos que iban desde la «Medicina, a la Aeronáutica, la Robótica y el Turismo» (Bower, Howe, McCredie, Robinson \& Grover, 2014); sin embargo, debemos reconocer que ha sido en los últimos años cuando está adquiriendo mayor significación y presencia, tanto en el sector industrial, como en el del ocio y el formativo (Cabero y García, 2016). Este aumento progresivo posiblemente se deba al incremento exponencial que en los últimos años han tenido algunos de los dispositivos o medios que suelen utilizarse para su manejo y observación, como son las tabletas y los celulares inteligentes. En este aspecto, recientemente Lagunes-Domínguez y Torres-Gastelú (2017), han realizado una investigación con estudiantes universitarios colombianos y mejicanos, que ha puesto de manifiesto que es una tecnología, la de los celulares, fuertemente disponible en los estudiantes, y respecto a la cual muestran un alto grado de acuerdo para su uso como tecnología de aprendizaje. 


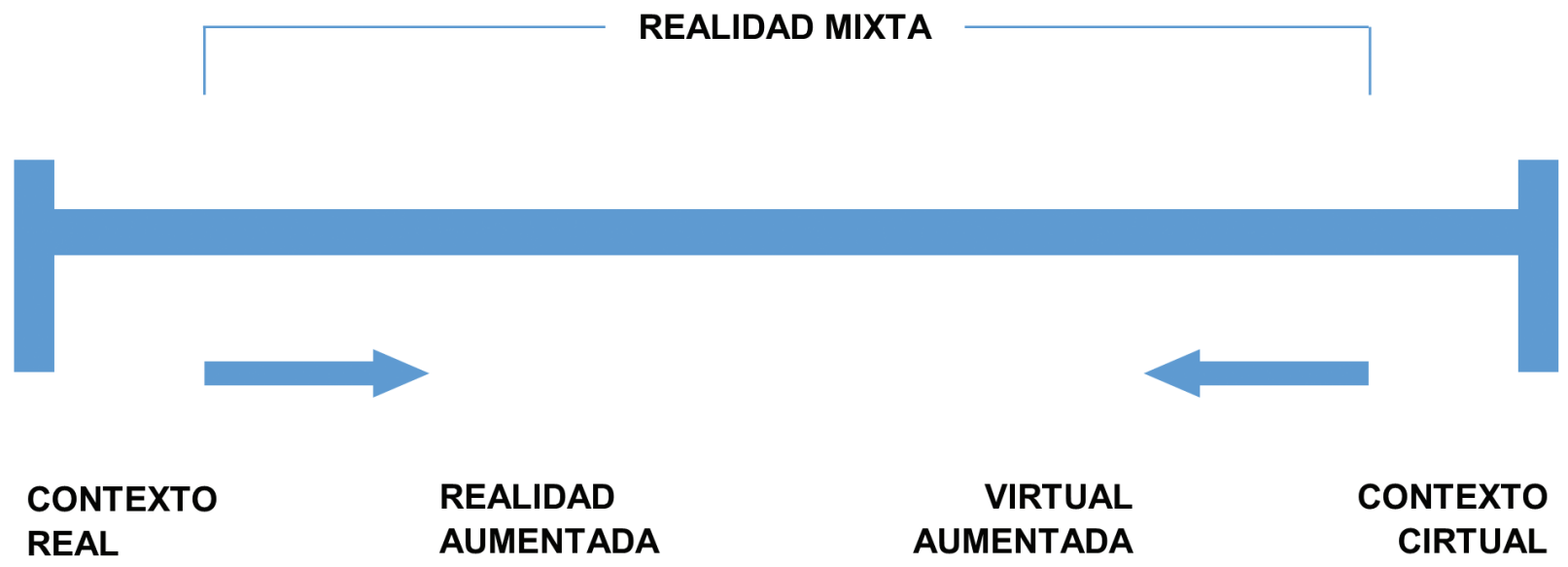

CONTINUUN REALIDAD—VIRTUALIDAD

Figura 1. Continuum realidad-virtualidad.

(Milgram, Takemura, Utsumi y Kishino, 1995).

A la hora de abordar su definición, una de las formas de hacerlo es contraponiendo lo que puede ser la realidad con la virtualidad (Milgram, Takemura, Utsumi y Kishino, 1995), según se puede apreciar en la figura 1, y desde esta perspectiva como señalan Cabero y García (2016) se puede indicar que «es una tecnología que permite la combinación de información digital e información física en tiempo real, por medio de distintos soportes tecnológicos, algunos muy tradicionales como los celulares y las webcams y otros más novedosos como las tabletas y las gafas de visión especiales, consiguiendo de esta forma una nueva realidad».

Otra forma de caracterizarla es contraponiéndola a la «Realidad Virtual» (RV), donde «no se combina la realidad con elementos informativos ubicados en los dispositivos tecnológicos para alcanzar con ello una nueva realidad, sino que en este caso el sujeto es ubicado en un contexto inmersivo tecnológico y no se ve mezclado con la realidad», según señalan Díaz, 2016; Johnson y Adams (2016). Con la primera, lo que se logra es combinar la realidad con lo virtual, con lo que el usuario puede accionar con ambos espacios: el físico y el digital; y la segunda, les facilita a los usuarios el acceso a una nueva galaxia alternativa de inmersión, la cual es trabajada en el ordenador donde ocurren diferentes tipos de experiencia sensoriales.

Lo anterior nos permite señalar que la RA y RV proponen formas diferentes de interaccionar con la realidad; mientras la RV traslada al sujeto a un mundo irreal, la RA lo que hace es añadir información al mundo real. De todas formas, no debemos olvidar que ya empiezan a existir experiencias de «Realidad Mixta» (RM), donde se combinan la RA y la RV, para obtener las máximas posibilidades que cada una de ellas nos ofrecen; o sea, se puede añadir un objeto irreal a un contexto real de formación, mediante una herramienta tecnológica, con lo que se favorece su contextualización en los procesos de formación.

Frente a otras tecnologías, la RA posee características específicas que la hace verdaderamente interesante y útil para la formación, tanto para la presencial, como para virtual y 
la mixta, entre las que según Barroso, Cabero, García, Calle, Gallego y Casado (2017), se reconoce «ser una realidad mixta; su integración coherente en tiempo real: ofrece diversidad de capas de información digital; su posibilidad de interacción; para la construcción del contenido debe intervenir la persona y enriquece o altera la información de la realidad con la información que se le integra».

\section{¿Qué posibilidades nos ofrece}

para su utilización educativa

y con qué dificultades nos

\section{encontramos para ser incorporada}

\section{a la formación?}

Por lo que respecta a sus posibilidades educativas, conviene percibirla desde el principio, como sugieren Johnson, Smith, Levine, y Haywood (2010), como una tecnología que podemos ver inmersa dentro de diferentes enfoques pedagógicos, y señalan, en concreto, los siguientes:

a. Aprendizaje constructivista: mediante el uso de RA se motiva a los estudiantes a involucrarse de una manera más profunda con las tareas, conceptos y recursos estudiados a través del uso de superposiciones de información, permitiendo establecer conexiones más significativas y duraderas en lo que al conocimiento se refiere.

b. El aprendizaje contextual: el aprendizaje auténtico y contextualizado se posibilita mediante la incorporación de experiencias educativas en el entorno del mundo real, así como la introducción del mundo real en el aula.

c. Aprendizaje basado en juegos: los sistemas de realidad aumentada pueden usarse para facilitar el aprendizaje basado en juegos inmersivos creando una narrativa digital, colocando a los estudiantes en un rol, proveyendo recursos auténticos e incorporando información contextualmente relevante.

d. Aprendizaje basado en la investigación: ofreciendo un medio para recopilar electrónicamente datos para análisis futuros y proporcionar modelos virtuales situados dentro de un contexto real fácilmente manipulable, así como proporcionando información que se considera contextualmente relevante para el tema que se está investigando. (Johnson, Smith, Levine, y Haywood, 2010).

A ellos, les incorporaríamos las propuestas del aprendizaje móvil y ubicuo; pero estos aspectos los retomaremos posteriormente.

Podemos ver, desde el principio, que es una tecnología que puede amoldarse a diferentes prácticas y experiencias educativas que pueden ir desde un aprendizaje objetivista; pues permite lograr, con su utilización, que los alumnos aprendan capacidades y protocolos concretos de actuación perfectamente definidos y estructurados; hasta posiciones constructivistas, donde los alumnos tengan que adoptar posiciones activas para la construcción del conocimiento y desde acciones donde la información se presenta, a través de estos objetos, en situaciones de aprendizaje basadas en el juego o en problemas. También como estudios de casos donde, mediante estos recursos, se les ofrezcan diferentes materiales para que los alumnos tengan que ofrecer una solución al problema diagnosticado. Por otra parte, no debemos olvidar que su uso propicia la contextualización de los conceptos al facilitar el conocimiento de la realidad.

Realizados estos comentarios, es oportuno presentar las posibilidades que una diversidad de autores le conceden a la RA (Wu, Lee, Chang y Liang, 2013; Carozza, Tingdahl, Bosché y Gool, 2014; Cubillo, Martín, Castro y Colmenar, 2014; Jeřábek, Rambousek y Wildová,2014; Barba, Yasaca y Manosalvas, 2015; Jamali, Fairuz, Wai y Oskam, 2015; Fonseca, Redondo y Valls, 2016; Han, Jo, Hyun y So, 2015; Santos, Wolde, Taketomi, Yamamoto, Rodrigo, Sandor, et al, 2016) y que se sintetizan a continuación:

a. Presentar la información esencial y de mayor valor, eliminando aquella que pueda obstaculizar su captación por el estudiante. 
b. Mostrar la realidad de forma enriquecida o aumentada de forma que se facilite su compresión por el estudiante.

c. Facilitar que el objeto sea observado desde diferentes puntos de vista, permitiendo que el estudiante pueda seleccionar la posición y el momento para su observación.

d. Garantizar que se potencie el u-learning o aprendizaje ubicuo.

e. Integrar el uso de simuladores o laboratorios virtuales, como «escenarios artificiales» que propicien un aprendizaje seguro y lo más cercano posible a la realidad.

f. Ofrecer a los estudiantes recursos documentales con información adicional en diferentes soportes que complementen el aprendizaje de los estudiantes.

g. Permitir la visualización de un objeto o fenómeno desde diferentes perspectivas, de forma que se potencie la inteligencia espacial de los estudiantes.

h. Propiciar que los estudiantes integren el uso de objetos de aprendizaje en RA a su uso cotidiano de forma que sean «proconsumidores» de estos medios.

i. Facilitar que expongan situaciones o fenómenos heterogéneos y temporales.

j. Garantizar la contextualización de la información ante cualquier situación.

Dentro de las posibilidades que ofrece la RA está el permitir acercarnos al contexto real, pero de manera que el alumno tenga que permanecer en él, y no se aisle, favoreciendo de esta forma un aprendizaje situado; pero a su vez, nos permite modificarlo y adaptarlo a las características y requerimientos cognitivos del estudiante; convirtiéndose en una tecnología inmersiva que permite crear y replicar situaciones interesantes para la formación del estudiante.

De todas maneras, no estaría mal, de cara a su utilización, no olvidarnos de una serie de principios, a la hora de la producción de estos objetos de aprendizaje:

-La tecnología de interacción condiciona; por tanto, desarrollar módulos con contenidos breves y directos, que no coadyuven a la desorientación cognitiva o al desbordamiento en el estudiante.

-Tener presente que su diseño sea flexible y lo más simple posible, como principio, no perdiendo de vista que los alumnos poseen diferentes características y capacidades cognitivas.

- Garantizar que permita interactuar con el objeto de forma intuitiva y fácil, de manera que sea tolerante con los errores y accesible para el usuario.

-Que se tienda a producir objetos multimedia, de forma que se propicie la activación de los diferentes tipos de inteligencias, al incorporar elementos de diversos sistemas simbólicos.

-Que se proyecte una construcción orientada a la acción, a partir de que permita que el sujeto interactúe con el objeto.

-Desarrollar una estructura multiplataforma.

-Y que no se pierda de vista su orientación hacia el aprendizaje móvil y situado.

Por otra parte, y como sugieren Pedraza, Amado, Lasso y Munévar (2017), la RA «defiende una perspectiva no lineal de la formación, lo que supone una adaptación a la nueva cultura hipertextual propia de las nuevas generaciones y facilita que los estudiantes naveguen, interactúen y construyan su propio conocimiento». Interacción hipertextual que, no olvidemos, favorece el desarrollo del pensamiento divergente y la potenciación de las inteligencias múltiples, pues conlleva la utilización de diferentes tipos de recursos y sistemas simbólicos.

En el terreno de la educación a distancia, la RA puede ser de gran ayuda para la obtención por el alumno de conocimientos prácticos a través de experimentos y laboratorios. Odeh, Shanab y Anabtawi (2015) llevan a cabo una investigación donde compararon laboratorios de RA, laboratorios virtuales y laboratorios tradicionales; encontrando que los primeros fueron bien aceptados por los estudiantes, fáciles de usar y de entender en su funcionamiento, y que 
creaban entornos muy similares a los laboratorios reales. Ello facilita la creación de prácticas educativas a estudiantes que llevan a cabo las acciones formativas a distancia, ya que permite la creación de experiencias dentro del entorno físico, permitiendo de esta forma una herramienta potente a los alumnos para la contextualización de la información.

Como señalan Han, Jo, Hyun, y So (2015), en primer lugar, «la tecnología RA permite a los estudiantes experimentar entornos de aprendizaje de inmersión por la manipulación de contenidos y objetos virtuales a través de una interfaz tangible»; y en segundo lugar, la tecnología RA puede «mejorar la comprensión de los alumnos o de los objetos complejos, mediante la presentación de una variedad de puntos de vista a través de tres dimensiones imágenes estereoscópicas y simulación virtual infundida con el entorno real; propiciando una transición suave de la mera presentación de la información para la construcción de exploración y conocimiento activo».

Frente a estas posibilidades no debemos olvidar que su incorporación educativa puede verse mermada por una serie de aspectos, independientemente de su novedad y la predisposición existente a ensalzar cualquier nueva tecnología que vaya apareciendo en el mercado, y que pudiera ser la "panacea» para resolver todos los problemas educativos. La historia de la Tecnología Educativa, está llena de futuros tecnológicos que nunca llegaron a ser presentes, sin olvidarnos de que innovar con las TIC ocurre cuando las movilizamos para alcanzar aprendizajes activos, abiertos, flexibles y colaborativos, y no solo por su presencialidad en las aula.

En el caso que analizamos nos encontramos con las siguientes dificultades:

-Se están desarrollando mayores tecnologías que experiencias educativas.

-La novedad con que se está desarrollando repercute en una falta de reflexión para su incorporación a la práctica educativa.
-Las experiencias de incorporación son más bien de acciones puntuales que de acciones planificadas y continuadas para su adopción educativa.

-Falta de materiales educativos para su introducción a la enseñanza.

-Carencia de acciones formativas para su utilización por parte de los docentes y sobre todo para su aplicación en estrategias innovadoras de formación.

-Los dispositivos tecnológicos que se utilizan para la interacción cuentan con diferentes sistemas operativos, lo que reclama la realización de diferentes versiones de los objetos.

-La dificultad y desorientación cognitiva que supone para algunos estudiantes el trabajar en un medio formado por la integración de lo real y de lo virtual.

-La casi nula investigación educativa.

-Y la falta de fundamentación teórica para tomar de decisiones respecto a su utilización y formas de hacerla.

A los anterior, podríamos incorporar las dificultades propias de las infraestructuras de los centros educativos, como son la falta de tecnología móvil para el trabajo en las aulas y la falta de conectividad; sin olvidarnos de la actitud recelosa en algunos centros y profesores, a permitir el uso de tecnología móvil en las aulas. Lógicamente, algunos de estos problemas no influyen en las acciones formativas a distancia.

Por lo que se refiere a la falta de fundamentación teórica, es cierto que una de las limitaciones que los docentes tienen para la utilización de cualquier tecnología es la inexistencia de un marco conceptual, de ahí que estén apareciendo nuevos modelos de capacitación de los docentes en las TIC, como el TPACK (Cabero y Barroso, 2016c) que indican que «ésta debe hacerse no solo desde una perspectiva tecnológica-instrumental, sino también pedagógica y de contenido». Pero centrándonos en el caso que nos ocupa de la RA, lo mismo que con cualquier tecnología, es no perder de vista que su fundamentación no debe hacerse únicamente apoyándonos en 
un único enfoque o teoría pedagógica, sino más bien en la combinación de varios de ellos, creando así un «Ecosistema de aprendizaje con RA». En este sentido, diferentes teorías están aportando pistas y sugerencias para su utilización, como la teoría de la disonancia cognitiva que se encuentra en relación con una visión constructivista del aprendizaje y fue formulada inicialmente por Festinger (1975); teoría que se refiere a la tensión con la que muchas veces se tropieza el sujeto cuando se enfrenta a dos pensamientos o situaciones que se encuentran en conflicto, bien por los conocimientos previos que posee la persona sobre ellos, o por sus creencias o actitudes.

Para resolver esta situación se requiere de una fuerte motivación y solventar así la disonancia. En este sentido, los objetos en RA pueden ofrecer diferentes perspectivas para adoptar y fundamentar la toma de una decisión. La teoría de la variación, desarrollada en sus comienzos por Mazur (1997), en la cual se expresa que las mejores y más enriquecedoras experiencias para el aprendizaje son aquellas «donde se pone en situación al estudiante para que tenga que experimentar o analizar para cambiar su concepción inicial». Ellas permiten a la RA ofrecer diferentes perspectivas de un fenómeno, o que interaccione con él desde diferentes planos y perspectivas. La teoría de la actividad que sugiere que la acción y la intervención del estudiante es una de las claves para la formación del aprendizaje; el aprendizaje visual, que nos indica que todo proceso de enseñanza aprendizaje en el que utilizamos un conjunto de organizadores gráficos e imágenes, ya sea para la representación de la información como para el trabajo con conceptos e ideas; ayudan al estudiante a transferir éstas, a pasar de lo abstracto a lo concreto, y a captar los elementos significativos de los conceptos. En este sentido la RA ayuda al estudiante a visualizar diferentes fenómenos desde diversas perspectivas que son determinadas por él en la interacción con el objeto; de igual forma el aprendizaje situado, que persigue que los estudiantes trabajen en los contextos reales donde se producen las situaciones, y que tiende a potenciar el aprendizaje colaborativo y grupal; y el enfoque constructivista de la enseñanza, que persigue entregar al estudiante determinadas herramientas que les permita abordar personalmente situaciones problemáticas que le faciliten aprender y mediante su solución seguir aprendiendo. Desde esta perspectiva, la RA propicia la participación directa de los alumnos en la construcción del conocimiento.

Por otra parte, no debemos olvidar que la experiencia con la RA favorece el aumento de la motivación y el desarrollo de prácticas altamente emocionantes, y ambas variables son determinantes para favorecer el desarrollo de la formación.

Para finalizar este apartado, presentaremos la propuesta realizada por Pedraza, Amado, Lasso y Munévar (2017), donde introducen un esquema de los diferentes enfoques, desde los que se puede abordar la utilidad educativa de la RA, y puede servirnos de síntesis de algunos de los comentarios que hemos realizado últimamente (Tabla 1).

\begin{tabular}{llll}
\hline ENFOQUE & INSTRUMENTAL & COGNITIVO & SISTÉMICO \\
\hline $\begin{array}{l}\text { Cooncepto } \\
\text { desde la RA }\end{array}$ & $\begin{array}{l}\text { Herramienta } \\
\text { y recurso }\end{array}$ & $\begin{array}{l}\text { Medición } \\
\text { pedagógica } \\
\text { con fines del } \\
\text { aprendizaje }\end{array}$ & $\begin{array}{l}\text { Modelado, } \\
\text { simulación } \\
\text { e inmersión }\end{array}$ \\
\hline $\begin{array}{l}\text { Modo de } \\
\text { asumir la RA }\end{array}$ & Actividad & Estrategia & Metodología \\
\hline
\end{tabular}

Tabla 1. Enfoques de aplicación de la RA

(Pedraza, Amado, Lasso y Munévar, 2017).

\section{Escenarios creados en RA}

Abordar la utilización educativa de la RA, es tener siempre presente el principio que su utilización está alcanzando a todos los niveles y disciplinas educativos.

Por lo que se refiere a los niveles educativos, nos encontramos con experiencias en primaria (Prendes, 2015; Villalustre y Moral, 
2016), secundaria-bachillerato-formación profesional (Bressler y Bodzin, 2013; De la Torre, Martín-Dorta, Saorín, Carbonel y Contero, 2013) y a nivel universitario (Rodríguez, 2013).

En el caso de las disciplinas, su utilización alcanza a diferentes áreas de estudio: Ingeniería y Arquitectura (De la Torre y otros, 2013), Urbanismo (Carozza, Tingdahl, Bosché y Gool, 2014), Matemáticas-Geometría (Coímbra, Cardoso y Mateus, 2015; Poot, Martin-González y Menéndez-Domínguez, 2016), Arte e Historia (Ruiz, 2011) aprendizaje de idiomas (Santos y otros, 2016; Hsu, 2017), Tecnología (Rodríguez, 2013), Diseño (Ko, Chang, Chen, y Hua, 2011), Química (Pasaréti, Hajdú, Matuszka, Jambori, Molnar y Turcsányi-Szabó, 2011), Ciencias Naturales (Fracchia, Alonso, y Martins, 2015), Biología (Solano, Casas, y Guevara, 2015), Geografía (Carbonell y Bermejo, 2017), Medicina (Barroso y Cabero, 2016b; Ferrer, Jiménez, Torralba y García, 2016) o Ciencias de la Educación (Garay, Tejada y Maíz, 2017; Garay, Tejada y Castaño, 2017).

En cierta medida podríamos decir que son nuestra imaginación y creatividad las que pueden limitar su implementación.

\section{Aportaciones desde la investigación para su utilización en la formación}

Aunque hemos señalado que uno de los problemas con que contamos para su incorporación es la falta de investigaciones; en los últimos años se han producido diferentes trabajos que están aportando ideas y pistas que nos permiten reflexionar sobre cómo debe ser su incorporación educativa, qué metodologías y estrategias podemos utilizar y cómo diseñar los objetos de aprendizaje en función de las características de sus destinatarios y los objetivos que se persigan.

Algunos de los resultados que nos encontramos son: «los alumnos muestran actitudes favorables hacia ella y su utilización aumenta la motivación hacia el aprendizaje» (Cózar, De Moya, Hernández y Hernández, 2015); que «favorecen la creación de un contexto constructivista de formación» (Chen y Tsai, 2012; Wojciechowski y Cellary, 2013); que «propicia un entorno activo de enseñanza»; (Fombona, Pascual y Madeira, 2012); que «despierta un elevado grado de satisfacción en los alumnos» (Han et al., 2015; Kim, Hwang y Zo, 2016), y que «su utilización mejora los resultados de aprendizajes» (Bongiovani, 2013; Chang, Wu y Hsu, 2013; Kamarainen et al, 2013).

Este apartado con los resultados de las investigaciones lo retomaremos posteriormente cuando abordemos los alcanzados por nosotros a través del proyecto de investigación RAFODIUN.

\section{Aportes a la utilización educativa desde el proyecto} de investigación RAFODIUN

Liderado por la Universidad de Sevilla (España) y financiada por el Ministerio de Economía y Competitividad del gobierno de España, desde 2015 se realiza el proyecto «Realidad Aumentada para Aumentar la Formación. Diseño, Producción y Evaluación de Programas de Realidad Aumentada para la Formación Universitaria (RAFODIUN) (EDU2014-57446-P)», que persigue los siguientes objetivos generales destinados a profundizar en las posibilidades educativas que puede ofrecer la RA en las universidades:

-Evaluar las posibilidades y potencialidades que ofrecen diferentes softwares utilizados para la creación de entornos tecnológicos bajo la arquitectura de la RA, para ser utilizados en contextos formativos universitarios.

- Analizar las posibilidades que los diferentes tipos de dispositivos de RA ofrecen para su aplicación en contextos de enseñanza universitaria.

-Diseñar y producir distintos contenidos en formato RA para ser aplicados en contextos de formación universitaria en distintas áreas curriculares, y evaluar sus posibilidades de cara al rendimiento de los alumnos. 
- Conocer el grado de motivación y nivel de satisfacción que despierta en los estudiantes universitarios el hecho de participar en experiencias formativas apoyadas en RA.

- Crear un entorno formativo bajo la arquitectura de la RA, en formato libro electrónico, para la capacitación del profesorado universitario en el diseño, producción y utilización educativa de la RA.

-Poner en acción y validar el entorno producido para la capacitación del profesorado universitario en el diseño, producción y utilización educativa de la RA.

-Conocer las posibilidades educativas que permiten que el alumno se convierta en productor de experiencias formativas apoyadas en la RA.

-Indagar sobre las dificultades técnicas, curriculares y organizativas que pudiera tener la RA para ser aplicada a los contextos de formación universitaria.

- Crear una comunidad virtual formada por profesorado universitario preocupado por el uso educativo de la RA. (Proyecto RAFODIUN, 2014).

El proyecto cuenta con un sitio web oficial (Figura 2), donde progresivamente se van presentando los diferentes hallazgos, objetos producidos y publicaciones realizadas.

La investigación se está desarrollando en diferentes fases y con distintos diseños de investigación, algunas ya finalizaron y cuentan con diferentes publicaciones que serán indicadas en su momento.

La primera fase, ya concluida, perseguía analizar el software de producción de la RA, para ello, nuestros expertos realizaron una evaluación que generó la selección de una lista de 35 programas. Los 10 software más conocidos fueron los siguientes: 1) Aurasma (84-88,77\%), 2) Layar (64-61,54\%), 3) ARToolkit, 4) Wikitude, 5) Vuforia, 6) Designers artoolkit, 7) Armedia, 8) Arlab, 9) Blippar, y 10) Arpa.

Programas que fueron evaluados de acuerdo con una serie de dimensiones, cuyos resultados se presentan, a continuación, en la Tabla 2.

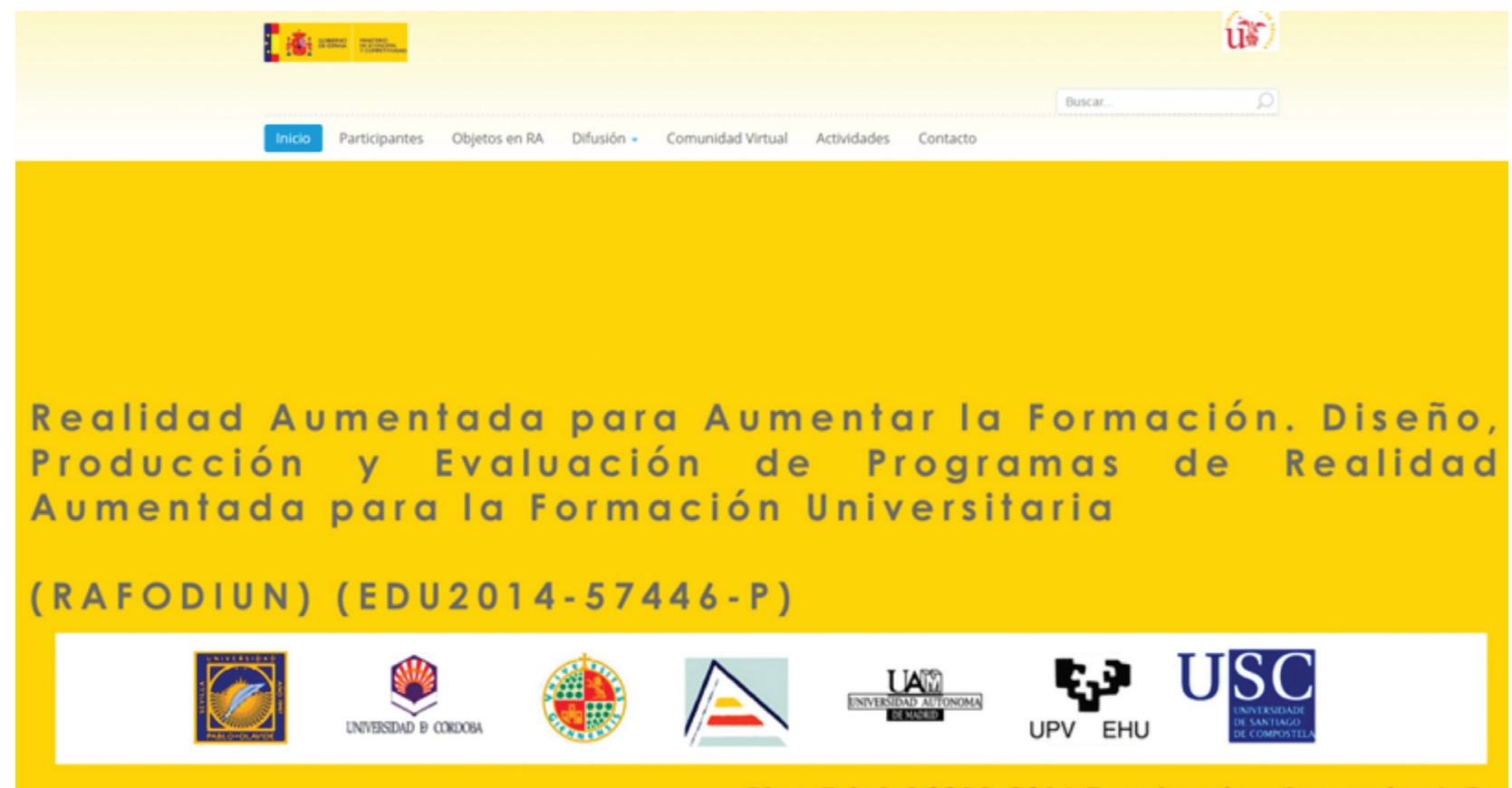

Plan Estatal 2013-2016 Excelencia - Proyectos I+D

Figura 2. Portal web del proyecto RAFODIUN.

(https://grupotecnologiaeducativa.es/proyectorafodiun/index.php). 


\begin{tabular}{lcccc} 
& $\begin{array}{c}\text { CONOCIMIENTO } \\
\text { TÉCNICO NECESARIO } \\
\text { MANEJAR } \\
\text { PROGRAMAS }\end{array}$ & $\begin{array}{c}\text { ACCESIBILIDAD/ } \\
\text { FACILIDAD } \\
\text { DE NAVEGACIÓN } \\
\text { DEL PROGRAMA }\end{array}$ & $\begin{array}{c}\text { POSIBILIDAD } \\
\text { DE INCORPORACIÓN } \\
\text { DE MATERIALES } \\
\text { DIFERENTES }\end{array}$ & $\begin{array}{c}\text { FACILIDAD } \\
\text { DE UTILIZACIÓN } \\
\text { POR DOCENTES } \\
\text { Y DISCENTES }\end{array}$ \\
\hline Arlab & 6.667 & 7.067 & 6.400 & 6.933 \\
\hline Armedia & 6.750 & 6.000 & 6.250 & 5.625 \\
\hline Arpa & 6.182 & 6.455 & 5.455 & 5.909 \\
\hline ARTTolkit & 7.033 & 6.267 & 7.000 & 5.867 \\
\hline Aurasma & 5.524 & 6.262 & 6.595 & 7.762 \\
\hline Blippar & 4.786 & 5.214 & 6.571 & 6.857 \\
\hline Designers artoolkit & 7.750 & 6.600 & 6.500 & 5.500 \\
\hline Layar & 6.063 & 6.344 & 6.813 & 6.531 \\
\hline Vuforia & 7.095 & 6.619 & 8.048 & 5.000 \\
\hline Wikitude & 5.800 & 5.920 & 6.040 & 6.920 \\
\hline
\end{tabular}

Tabla 2. Valoración del software de producción de objetos de RA de acuerdo con una serie de dimensiones. (Elaboración propia).

Otra de las fases consistió en crear una comunidad virtual con profesores e investigadores preocupados por la utilización en los procesos de enseñanza aprendizaje de la RA, y para ello se creó una comunidad virtual cerrada en el entorno de Google+ (Figura 3). Hay que indicar que su carácter cerrado solo implica que la persona que desee participar en ella lo único que debe hacer es solicitar su incorporación y es admitida.

La comunidad está conformada por 542 miembros de diferentes países latinoamericanos y en el último estudio realizado, generaron los siguientes datos interesantes. El número de post que se realizaron en la comunidad virtual en el tiempo analizado fue de 457:219 post, que se
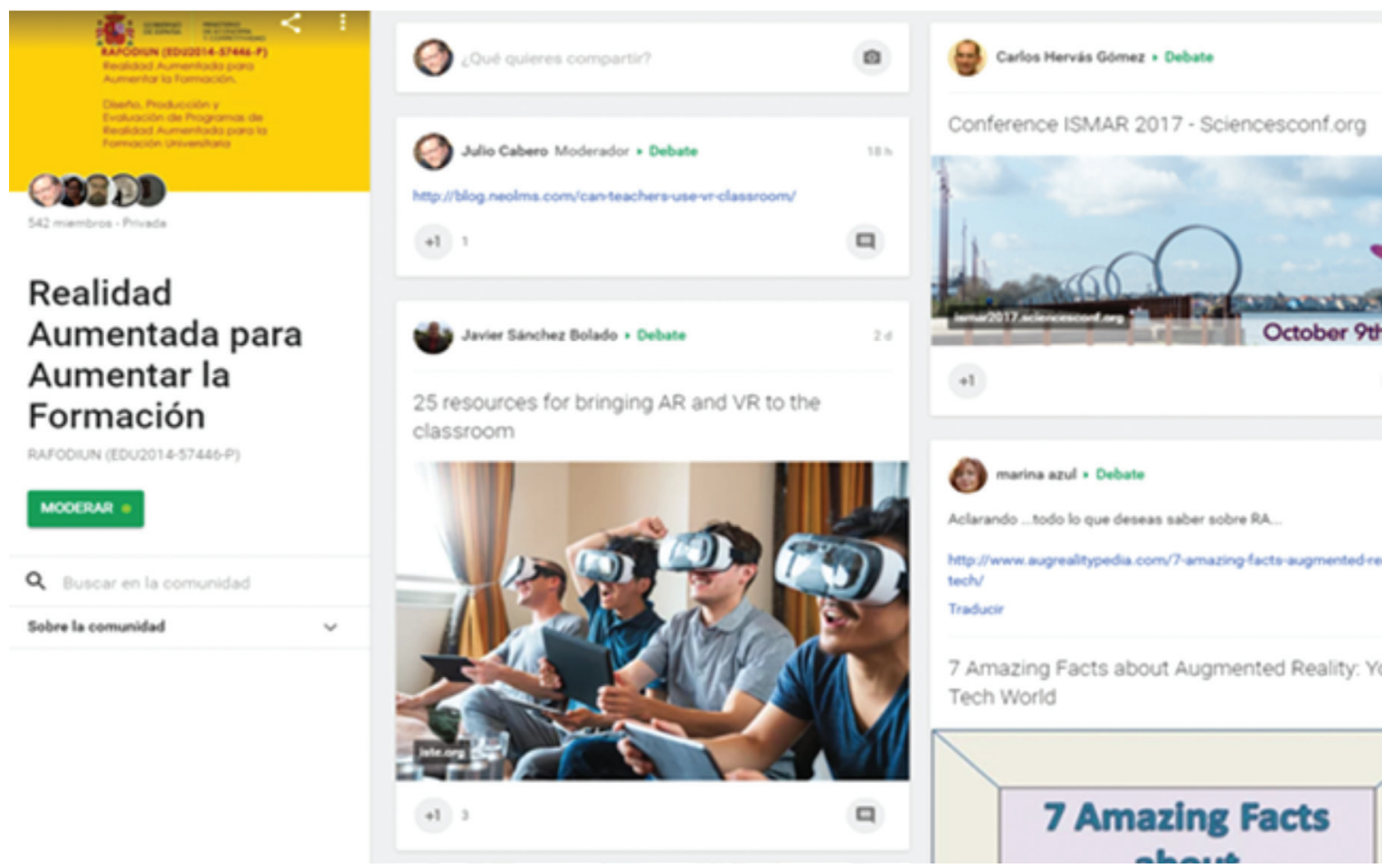

\section{Realidad \\ Aumentada para \\ Aumentar la Formación}

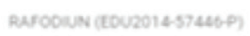

\section{moceresa :}

a

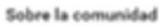

Figura 3. Portal comunidad virtual RAFODIUN.

(https://plus.google.com/u/0/communities/102143147822806126247). 
efectuaron en horario de la mañana y 238 en la tarde/noche, siendo la media de envíos por día de 1.47. Los días con mayor número de post enviados a la CV fueron: 5 de noviembre de 2015 (811), 4 de enero de 2016 (810), 5 de diciembre de 2015 (8), 27 de marzo de 2016 (7), y 13 de febrero; 1 y 31 de marzo de 2016 con 6 respectivamente. Los meses con mayor número de publicaciones han sido los de enero (63) y mayo (73) de 2016, y octubre (53) y noviembre (60) de2015. De los 478 miembros en la red existentes en aquel momento, solo se puede considerar a 78 como «activos», Se entiende por «activos» a aquellos que bien han iniciando un post o han intervenido en el mismo. De estas personas activas, $42(53.85 \%)$ eran hombres y $36(46.15 \%)$ mujeres. Lo verdaderamente significativo de la comunidad es que sigue activa, las personas interesadas y que forman parte de ésta; pueden recibir desde experiencias que se están llevando a cabo, publicaciones, así como nuevos software $\mathrm{y}$ hardware aparecidos.
La $3^{\text {a }}$ fase de RAFODIUN fue dedicada al diseño y desarrollo de objetos de aprendizaje para las distintas disciplinas académicas universitarias que van desde la medicina, las ciencias de la educación, hasta las bellas artes, entre otras. Es necesario indicar que estos objetos producidos iban desde los que podríamos clasificar como de nivel I (trabajamos un patrón cuadro como lanzador-activador del objeto), del nivel II (utilizamos una imagen plana de realidad como lanzador), de nivel III (el lanzador es un objeto o un escenario 3D). Estos objetos incluían diferentes recursos de tipo vídeo (con o sin canal alfa -fondo transparente o sin transparencia), animación $3 \mathrm{D}$, documentos PDF o HTML, imágenes, vídeos en $360^{\circ}$, e inserción de audio.

Los objetos producidos se pueden observar tanto en la página web del proyecto, (Figura 4), como en la del Secretariado de Recursos Audiovisuales de la Universidad de Sevilla.

Dichos contenidos, una vez producidos, fueron utilizados en secciones de clase por sus

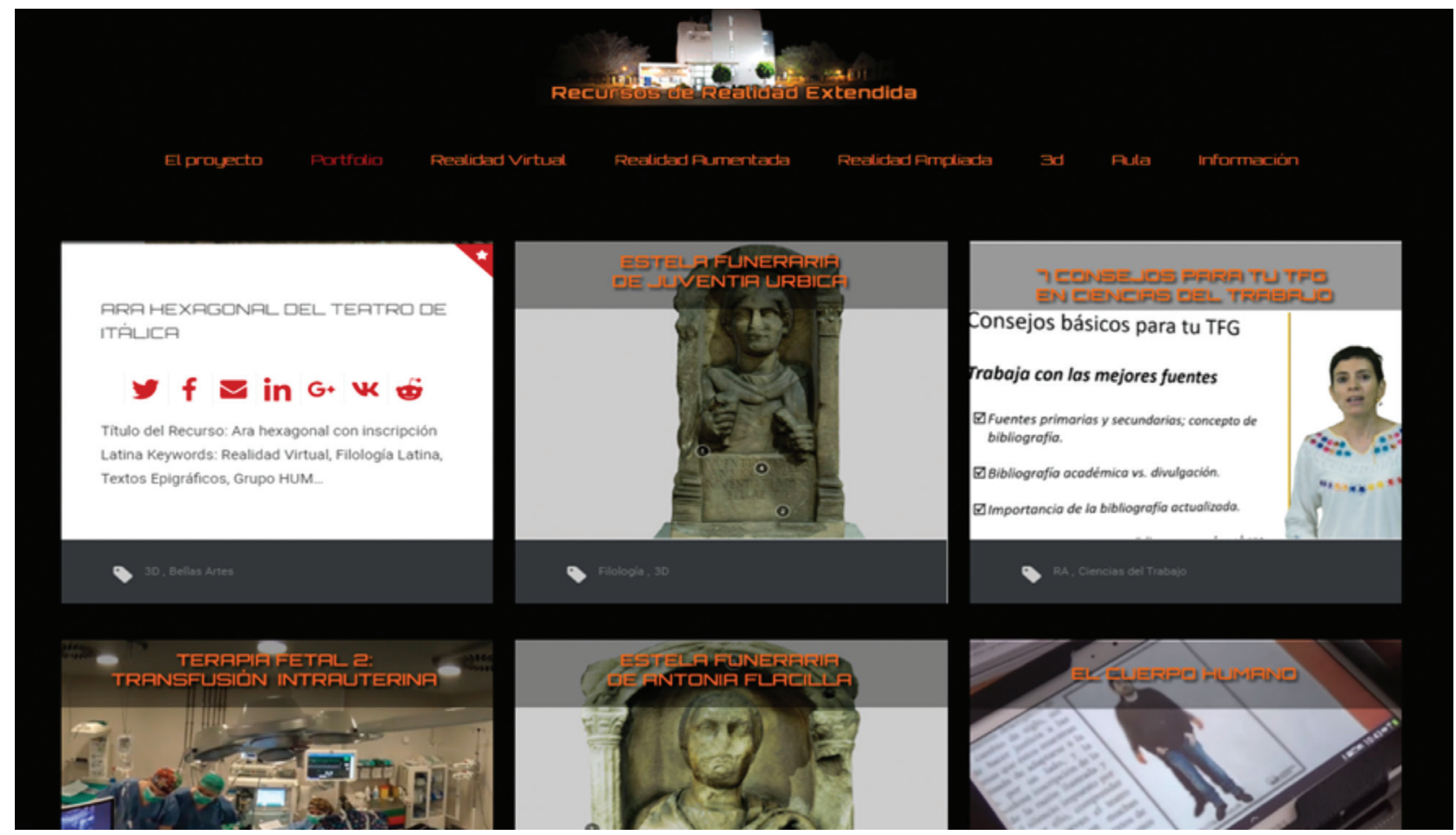

Figura 4. Portal de RA del SAV de la Universidad de Sevilla. (http://intra.sav.us.es/proyectorafodiun/index.php/objetos-en-ra). 
profesores, y se analizaron diferentes aspectos, como el grado de satisfacción o aceptación que despertaba la utilización de la tecnología medida mediante el «Modelo de Aceptación de Tecnología» (TAM) de Davies, Bagozzi, y Warshaw (1992); la motivación despertada por su utilización analizada, con la aplicación de la «Encuesta de Motivación para Materiales de Instrucción, (IMMS)» de Keller (2010); el rendimiento adquirido en la interacción con la tecnología, y la evaluación de la calidad técnica y didáctica de los materiales producidos por los estudiantes. Los resultados encontrados han sido expuestos en diferentes publicaciones: Barroso, Cabero y Moreno (2016); Barroso y Cabero (2016a); Cabero y Barroso (2016a); Gallego (2016); Fernández (2016 y 2017); Cabero, Fernández y Marín (2017); Cabero, Llorente, y Gutiérrez-Castillo (2017); Garay, Tejada y Castaño (2017); Garay, Tejada y Maíz (2017) y Barroso, Gutiérrez, Llorente y Valencia (2019). En la página web del proyecto se publican las diversas producciones que vayan surgiendo.

En líneas generales podemos señalar las siguientes conclusiones obtenidas:

Los alumnos muestran un alto nivel de satisfacción cuando interaccionan con los objetos en RA, sin importar el tipo de objeto utilizado, la diversidad de recursos que incorporaba y los de la disciplina.

-Los estudiantes evidencian un gran nivel de motivación y aceptación de las tecnologías o medios cuando interaccionan con objetos de Realidad Aumentada, independientemente del tipo de objeto utilizado, de la diversidad de recursos que incorporaba y los de la disciplina.

-Los objetos producidos fueron evaluados positivamente, tanto por profesores como por estudiantes, respecto a su calidad técnica y didáctica.

-Los alumnos que interaccionaron con los objetos adquirieron los contenidos por ellos presentados.

-En líneas generales, tanto profesores como alumnos no encontraron mucha dificultad para la incorporación de estos objetos en la enseñanza y para su manejo técnico. (Cabero y Marín-Díaz, 2018; Cabero Vázquez-Cano, López-Meneses, 2018; Martínez y Fernández, 2018; Cabero, Batanero y Barroso, 2019; Cabero, Barroso y Llorente, 2019; Cabero y Roig, 2019; Cabero, Vázquez-Cano, López, Jaén Martínez, 2020).

Hay que indicar que algunos de estos objetos producidos se realizaron bajo la modalidad de «materiales impresos enriquecidos con RA» (Ferrer, Jiménez, Torralba y García, 2016; Nadolny, 2016) (Figura 5), y que han supuesto una experiencia significativa para los estudiantes.

Nuestra 4ta fase del proyecto se refería a conocer las posibilidades educativas de la RA, para que el estudiante se transforme en creador de experiencias formativas con el apoyo de esta herramienta. En este caso, la experiencia se realizó con estudiantes de Pedagogía, de
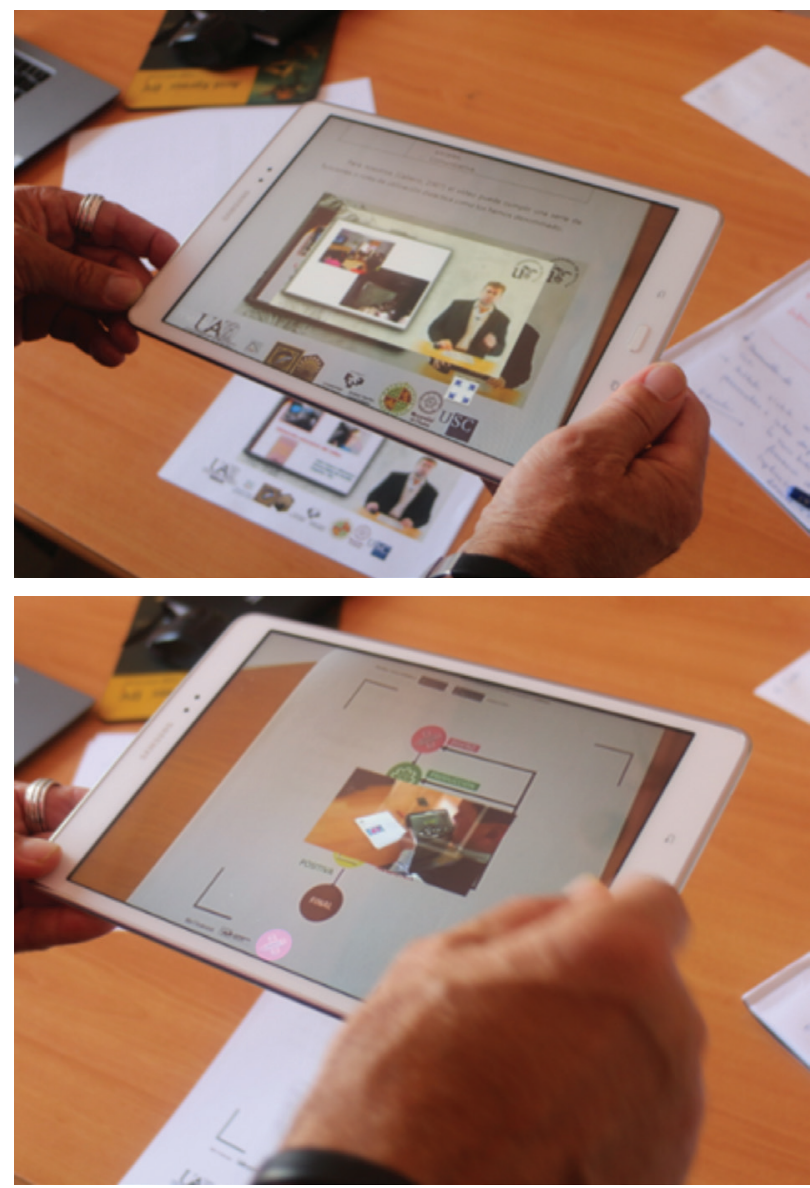

Figura 5. Objetos producidos. (Elaboración propia). 
Educación Infantil, y de Educación Primaria; que cursaban las asignaturas de «Tecnología Educativa» y «Tecnologías de la Información y Comunicación aplicadas a la Educación», y estuvo orientada a la producción de objetos por parte de éstos, que lleva a movilizar el aprendizaje de orden superior. Recuérdese que, en la taxonomía de Bloom para la era digital, la última categoría ya no es la evaluación sino la creación de objetos y acciones de aprendizaje. Los resultados encontrados, nos han llevado a señalar que los estudiantes son capaces de producir estos objetos, y que tales producciones los llevan a adquirir un elevado grado de aceptación de la tecnología y de motivación para el aprendizaje (Barroso y Gallego, 2017a y b).

Nuestra última fase, y es en la que nos encontramos actualmente, se enfoca a la producción de un material impreso en la modalidad de «libro enriquecido con objetos de RA», para la formación de los docentes en su utilización educativa. El material se articula alrededor de seis puntos, donde se aborda la conceptualización, tipología, fases o etapas para la producción de objetos de aprendizaje en RA y los diferentes

\section{Referencias}

Barba, R., Yasaca, S. y Manosalvas, C. (2015). Impacto de la realidad aumentada móvil en el proceso enseñanza-aprendizaje de estudiantes universitarios del área de medicina. Investigar con y para la Sociedad. 3., Cádiz: Bubok Publishing. pp.1421-1429.

Barroso, J. y Cabero, J. (2016a). El diseño de una investigación: el proyecto RAFODIUN. RIITE. Revista Interuniversitaria de Investigación en Tecnología Educativa. 1, pp.12-25.

(2016b). Evaluación de objetos de aprendizaje en realidad aumentada: Estudio piloto en el grado de medicina. Enseñanza y Teaching, 34(2), pp.149-167. https://doi. org/10.14201/et2016342149167

Barroso, J. y Gallego, O. (2017a). La realidad aumentada y su aplicación en la educación superior. Revista del Salomé. 1(29), pp.111-124.

—. (2017b). Producción de recursos de aprendizaje apoyados en realidad aumentada por parte de los estudiantes productos que se pueden obtener, en torno a aplicaciones móviles, software y sistemas que permiten su producción y visualización, así como sus usos educativos.

Durante el desarrollo del presente estudio, en sus diferentes fases, también se han identificado una serie de obstáculos que deben ser superados a nivel académico por las universidades: «falta de teorías educativas para explicar su utilización, formación del profesorado, limitadas investigaciones para su utilización, y poca flexibilidad de los currículums»». (Barroso, J., Gutiérrez, J.J., Llorente, M. d. C., y Valencia, 2019).

Para finalizar, se deben reiterar dos aspectos comprobados:

1. La RA no se puede utilizar como sustituto de la enseñanza; se debe usar como una herramienta tecnológica que la complemente, y

2. Su utilización debe insertarse dentro del proceso de enseñanza aprendizaje para garantizar su efectividad, mediante el diseño de unos objetivos muy precisos, donde se establezca la forma en que serán utilizados por parte de los estudiantes, evitando, en todo momento, caer en la atracción que las tecnologías despiertan.

de magisterio. Edmetic. Revista de Educación Mediática y TIC. 6(1), pp.23-38.

Barroso, J., Cabero, J. y Moreno, A.M. (2016). La utilización de objetos de aprendizaje en realidad aumentada en la enseñanza de la medicina. Innoeduca. International Journal of Technology and Educational Innovation. 2(2), pp.77-83.

Barroso, J., Cabero, J., García, F., Calle, F., Gallego, O., Casado, I. (2017). Diseño, producción, evaluación y utilización educativa de la Realidad Aumentada. Sevilla; Secretariado de Recursos Audiovisuales y Nuevas Tecnologías. Universidad de Sevilla.

Barroso, J., Gutiérrez, J.J., Llorente, M. d. C. y Valencia, R. (2019). Difficulties in the Incorporation of Augmented Reality in University Education: Visions from the Experts. Journal of New Approaches in Educational Research. 8(2), pp.126-141. Recuperado de https://doi. org/10.7821/naer.2019.7.409 
Bongiovani, P. (2013). Realidad aumentada en la escuela: Tecnología, experiencias e ideas.Educ@conTIC. Recuperado de http://www.educacontic.es/blog/realidad

Bower, M., Howe, C., McCredie, N., Robinson, A., \& Grover, D. (2014). Augmented Reality in education-cases, places and potentials. Educational Media International. 51(1), pp.1-15.

Bressler, D.M. y Bodzin, A.M. (2013). A mixed methods assessment of students' flow experiences during a mobile augmented reality science game. Journal of Computer Assisted Learning, 29(6), pp.505-517.

Cabero, J. (2016). La gestión del conocimiento en las organizaciones corporativas. En Gairín, J. (ed). Aprendizaje situado y aprendizaje conectado: implicaciones para el trabajo. Madrid: Wolters Klumer, pp.61-75.

Cabero, J. y Barroso, J. (2016a). The educational possibilities of augmented reality. NAER. New Approaches in Educational Research. 5(1), pp.44-50.

- (2016c). ICT teacher training: A view of the TPACK model / Formación del profesorado en TIC: Una visión del modelo TPACK. Cultura y Educación. 28(3), pp.633-663.

Cabero, J. y García, F. (2016). Realidad Aumentada. Tecnología para la formación. Madrid: Síntesis.

Cabero, J. y Marín-Díaz, V. (2018). Blended Learning y Realidad Aumentada: Experiencias de diseño docente. RIED. Revista Iberoamericana de Educación a Distancia. 21(1), pp.54-74.

Cabero, J., Vásquez-Cano, E., López, E., Jaén, A. (2020). Posibilidades formativas de la tecnología aumentada. Un estudio diacrónico en escenarios universitarios. $R e$ vista Complutense de Educación. 31(2), pp.143-154.

Cabero, J. y Roig, R. (2019). The motivation of technological scenarios in augmented reality (AR): Results of different experiments. Applied Sciences. 9. https://doi. org/10.3390/app9142907.

Cabero, J., Barroso, J. y Llorente, M.C. (2016). Technology acceptance model y realidad aumentada: Estudio en desarrollo. Revista Lasallista de Investigación. 13(2), pp.18-26.

—. (2019). La realidad aumentada en la enseñanza universitaria. REDU. Revista de docencia universitaria. 17(1), pp.105-118

Cabero, J., Batanero, J.M. y Barroso, J. (2019). Adoption of augmented reality technology by university students. Heliyon. 5. e01597. Recuperado de https://grupotecnologiaeducativa.es/images/bibliovir/RA.pdf

Cabero, J., Fernández, B. y Marín, V. (2017). Dispositivos móviles y realidad aumentada en el aprendizaje del alumnado universitario. Revista Iberoamericana de Educación a Distancia. 20(2), Recuperado de http://revistas.uned. es/index.php/ried/article/view/17245/15664

Cabero, J., Llorente Cejudo, M.C., y Gutiérrez-Castillo, J.J. (2017). Los alumnos como evaluadores de objetos de aprendizaje en Realidad Aumentada. RED. Revista de Educación a Distancia. 53, artículo 4.

Cabero, J., Vázquez-Cano, E., López-Meneses, E. (2018). Uso de la Realidad Aumentada como recurso didáctico en la Enseñanza Universitaria. Formación Universitaria. 11(1), pp.25-34.

Carbonell, C. y Bermejo, L. (2017). Landscape interpretation with augmented reality and maps to improve spatial orientation skill. Journal of Geography in Higher Education. 41(1), pp.119-133.

Carozza, L., Tingdahi, D., Bosché, F., y Gool, L. (2014). Markerless vision-based augmented reality for urban planning. Computer-Aided Civil and Infrastructure Engineering. 29(1), pp.2-17.

Chang, H., Wu, K. y Hsu, Y. (2013). Integrating a mobile augmented reality activity to contextualize student learning of a socioscientific issue. British Journal of Educational Technology. 44(3), E95-E99.

Chen, C.-M., y Tsai, Y. (2012). Interactive augmented reality system for enhancing library instruction in elementary schools. Computers y Education. 59, pp.638-652.

Coimbra, M., Cardoso, T. y Mateus, A. (2015). Augmented Reality: An enhancer for higher education students in math's learning? Procedia Computer Science. 67, pp.332-339. doi: 10.1016/j.procs.2015.09.277

Cózar, R., De Moya, M., Hernández, J., y Hernández, J. (2015). Tecnologías emergentes para la enseñanza de las Ciencias Sociales. Una experiencia con el uso de Realidad Aumentada en la formación inicial de maestros. Digital Education Review. 27, pp.138-153.

Cubillo, J., Martín, S., Castro, M. y Colmenar, A. (2014). Recursos digitales autónomos mediante realidad aumentada. Revista Iberoamericana de Educación a Distancia. 17, pp.241-274.

Davis, F.D.; Bagozzi, R.P.; Warshaw, P.R. (1992). Extrinsic and intrinsic motivation to use computers in the workplace. Journal of Applied Social Psychology. 22 (14), pp.1.111-1.132.

De la Torre Cantero, J., Martín-Dorta, N., Saorín Pérez, J.L., Carbonell Carrera, C., y Contero González, M. (2013). Entorno de aprendizaje ubicuo con realidad aumentada y tabletas para estimular la comprensión del espacio tridimensional. RED, Revista de Educación a Distancia 37. Recuperado de http://www.um.es/ead/red/37

Díaz, M. (2016). Augmented reality versus virtual reality: The battle is real. Techcrunch. Recuperado de http:// 
techcrunch.com/2016/01/04/ar-vs-vr-the-battle-isreal/?ncid=rss

Fernández, B. (2016). Aplicación del modelo de aceptación tecnológica (TAM) al uso de la Realidad Aumentada en estudios universitarios de educación primaria. En Roig-Vila, R. (ed). Educación y Tecnología. Propuestas desde la investigación y la Innovación educativa. Barcelona: Octaedro, pp.91-92.

(2017). Factores que influyen en el uso y aceptación de objetos de aprendizaje de realidad aumentada en estudios universitarios de Educación Primaria. Edmetic. Revista de Educación Mediática y TIC. 6(1), pp.203-219.

Ferrer, J., Jiménez, M.A., Torralba, J., y García, M. (2016). La realidad aumentada. Nuevas tecnologías en la formación de graduados en podología. Desarrollo y evaluación. En A. Allueva y J.L. Alejandre (Coords.). Simbiosis del aprendizaje con las tecnologías. (147-160), Zaragoza, España: Universidad de Zaragoza.

Festinger, L. (1975). Teoría de la disonancia cognitiva. Madrid: Instituto de Estudios políticos.

Fombona, J., Pascual, M.J. y Madeira, M.F. (2012). Realidad aumentada, una evolución de las aplicaciones de los dispositivos móviles. Píxel-Bit. Revista de Medios y Educación. 41, pp.197-210.

Fonseca, D., Redondo, E. y Valls, F. (2016). Motivación y mejora académica utilizando realidad aumentada para el estudio de modelos tridimensionales arquitectónicos. Education in the Knowledge Society, EKS. 17(1), pp.45-64.

Fracchia, C., Alonso, A., Martins, A. (2015). Realidad aumentada aplicada a la enseñanza de Ciencias Naturales. TE \& ET. 16, pp.7-15. Recuperado de http://sedici. unlp.edu.ar/handle/10915/50745

Gallego, O. (2016). Satisfacción de los estudiantes del grado de magisterio de la Universidad de Sevilla con el uso de la Realidad Aumentada para la creación de contenidos educativos. En Roig-Vila, R. (ed). Educación y Tecnología. Propuestas desde la investigación y la Innovación educativa. Barcelona: Octaedro, pp.310-311.

Garay, U., Tejada, E. y Castaño, C. (2017). Percepciones del alumnado hacia el aprendizaje mediante objetos educativos enriquecidos con realidad aumentada. Edmetic. Revista de Educación Mediática y TIC. 6, 1, pp.145-164.

Garay, U., Tejada, E. y Maíz, I. (2017). Valoración de objetos educativos enriquecidos con realidad aumentada: una experiencia con alumnado de máster universitario. Pixel-Bit. Revista de Medios y Educación. 50, pp.19-31.

Han, J., Jo, M., Hyun, E. \& So, H. (2015). Examining young children's perception toward augmented reality-infused dramatic play. Education Tech Research Development. 63. pp.455-474. https://doi.org/10.1007/s11423-015-9374-9
Hsu-T-C. (2017). Learning English with Augmented Reality: Do learning styles matter? Computers \& Education. 106, pp.137-149.

Jamali, S., Fairuz, M. Wai, K. \& Oskam, Ch. (2015). Utilising mobile-augmented reality for learning human anatomy. Procedia - Social and Behavioral Sciences. 197, pp.659-668.

Jeřábek, T., Rambousek, V. \& Wildová, R. (2014). Specifics of Visual Perception of The Augmented Reality in The Context of Education. Procedia - Social and Behavioral Sciences. 159, pp.598-604.

Johnson, L., Smith, R., Levine, A. \& Haywood, K. (2010). NMC Horizon Report: 2010 K-12 Edition. Austin, Texas: The New Media Consortium. Recuperado de https://www.learntechlib.org/p/182020/.

Johnson, L., Adams, S., Cummins, M., Estrada, V., Freeman, A. \& Hall, C. (2016). NMC Horizon Report: 2016 Higher Education Edition. Austin, Texas: The New Media Consortium.

Johnson, L. \& Adams, S., (2016). NMC Horizon Report: 2016 Higher Education Edition. Austin, Texas: The New Media Consortium.

Kamarainen, A., Metcalf, Sh., Grotzer, T., Browne, A., Mazzuca, D., Tutwiler, M., \& Dede, Ch. (2013). EcoMOBILE: Integrating augmented reality and probeware with environmental education field trips. Computers $y$ Education. 68, pp.545-556.

Keller, J.M. (2010). Motivational design for learning and performance. New York: Springer Science+Business.

Kim. K., Hwang, J. \& Zo, H. (2016): Understanding users' continuance intention toward smartphone augmented reality applications. Information Development. 32(2), pp.161-174.

Ko, Ch-H., Chang, T., Chen, Y. \& Hua, L. (2011). The Application of Augmented Reality to Design Education. En M. Chang et al. (Eds.). Edutainment Technologies. Educational Games and Virtual Reality / Augmented Reality Applications (pp.20-24). Heidelberg Berlín: Springer.

Lagunes-Domínguez, A. y Torres-Gastelú, C., (2017). Prospectiva hacia el aprendizaje móvil en estudiantes universitarios. Formación univesitaria. 10, 1.

Martínez, S. y Fernández, B. (2018). Objetos de Realidad Aumentada: percepciones del alumnado de Pedagogía. PixelBit. Revista de Medios y Educación. 53, pp.207-220.

Mazur, E. (1997). Peer Instruction: A User's Manual. New Jarse: Prentice Hall.

Milgram, P., Takemura, H., Utsumi, A. \& Kishino, F. (1995). Augmented reality: a class of displays on the reality-virtuality continuum. Telemanipulator and Telepresence 
Technologies. 2351. pp.282-292. doi: https://doi. org/10.1117/12.197321

Nadolny, L. (2016). Interactive print: The design of cognitive tasks in blended augmented reality and print documents. British Journal of Educational Technology. 48(3), pp.1-10. https://doi.org/10.1111/bjet.12462

Odeh, S., Shanab, S. y Anabtawi, M. (2015). Augmented Reality Internet Labs versus its Traditional and Virtual Equivalence. iJET. 10(3), pp.4-9.

Pasaréti, O., Hajdin, H., Matusaka, T., Jámbori, A., Molnár, I. \& Tucsányi-Szabó, M. (2011). Augmented reality in education. INFODIDACT 2011 Informatika Szakmódszertani Konferencia. Recuperado de http://people.inf.elte.hu/tomintt/infodidact 2011.pdf

Pedraza, C., Amado, O., Lasso, E. y Munévar, P. (2017). La experiencia de la realidad aumentada (RA) en la formación del profesorado en la universidad nacional abierta y a distancia UNAD- Colombia. Pixel-Bit. Revista de Medios y Educación. 51, pp.111-13. doi: https://doi.org/10.12795/pixelbit.2017.i51.08

Poot, A., Martin-González, A., Menéndez-Domínguez, V. (2016). Aprendizaje de vectores euclidianos utilizando un sistema de realidad aumentada. Research in Computing Science. 89, pp.9-16.

Prendes, C. (2015). Realidad aumentada y educación: análisis de experiencias prácticas. Pixel-Bit. Revista de Medios y Educación. 46, pp.187-203.

Realidad Aumentada para Aumentar la Formación. Diseño, Producción y Evaluación de Programas de Realidad Aumentada para la Formación Universitaria. Proyecto RAFODIUN (2014). Recuperado de https://grupotecnologiaeducativa.es/proyectorafodiun/index.php
Rodríguez, M. (2013). Experimentando la realidad aumentada. Integrando tecnología en el salón de clase. Recuperado de http://mbintegrandotecnologia.blogspot.com/2013/04/ experimentando-la-realidad-aumentada.html

Ruiz, D. (2011). La realidad aumentada y su dimensión en el arte: La obra aumentada. Arte y Políticas de Identidad. 5, pp.129-144.

Santos, M. Wolde, A., Taketomi, T., Yamamoto, G., Rodrigo, M., Sandor, Ch.\& Kato, H. (2016). Augmented reality as multimedia: the case for situated vocabulary learning. Research and Practice in Techology Enhanced Learning. 11(4), pp.1-23.

Solano, C.A., Casas J.F. y Guevara, J.C. (2015). Aplicación móvil de realidad aumentada para la enseñanza de la clasificación de los seres vivos a niños de tercer grado. Ingeniería. 20(1), pp.101-115.

Susskind, R. y Suukind, D. (2016). El futuro de las profesiones. Cómo la tecnología transformará el trabajo de los expertos humanos. España: Teell.

Tecnológico de Monterrey (2015). Reporte EduTrends. Radar de Innovación Educativa 2015. Monterrey: Tecnológico de Monterrey.

Villalustre, L. y Del Moral, M.E. (coords.) (2016). Experiencias interactivas con realidad aumentada en las aulas. Barcelona: Octaedro.

Wojciechowski, R. \& Cellary, W. (2013). Evaluation of learners' attitude toward learning in ARIES augmented reality environments. Computers y Education. 68, pp.570-585.

Wu, H-S., Lee, S., Chang, H-Y. \& Liang, J. (2013). Current status, opportunities and challenges of augmented reality in education. Computers y Education. 62, pp.41-49.

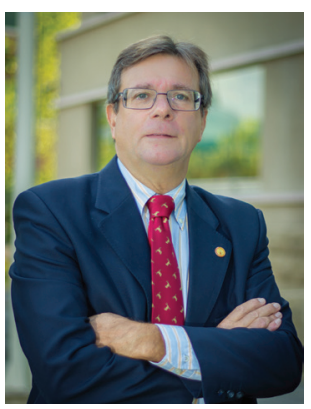

\section{Julio Cabero Almenara}

Doctor en Ciencias de la Educación. Catedrático de Tecnología Educativa de la Universidad de Sevilla (España), director del Secretariado de Recursos Audiovisuales de la US. Director del Grupo de Investigación Didáctica de la Junta de Andalucía. Director de la Revista: «Píxel-Bit. Revista de Medios y Educación. Cuatro sexenios de investigación reconocidos por el Ministerio de Educación (Gobierno de España). Ha dirigido más de 51 tesis doctorales y diferentes proyectos de investigación de convocatorias públicas: Proyectos de Excelencia, Estudios y Análisis, I+D+i. Ha recibido premios y distinciones nacionales e Internacionales (Venezuela, R. Dominicana y México) y está actualmente en el ranking de investigadores españoles con el \# 213. 


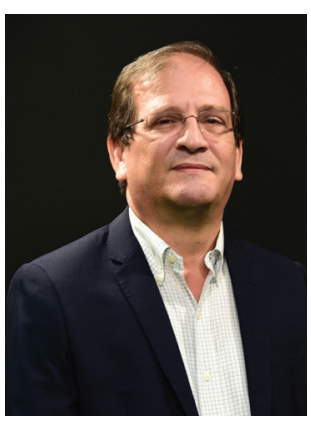

\section{Angel Puentes Puente}

Doctor por la Universidad de Sevilla. Máster en Tecnología Computacional para la Educación y Licenciado en Educación, Especialidad Física y Astronomía en la Universidad Pedagógica de Pinar del Río, Cuba. Docente en la PUCMM, UNPHU y UFHEC. Miembro de Asociación EDUTEC. Ha participado en grupos de investigación internacionales sobre la Educación a Distancia, Competencias Digitales de los docentes y estudiantes, las redes sociales en los procesos educativos, Realidad Aumentada, entre otros. Es autor y coautor de varias publicaciones en el área de la Física Educativa, Educación a Distancia y Tecnología Educativa. 


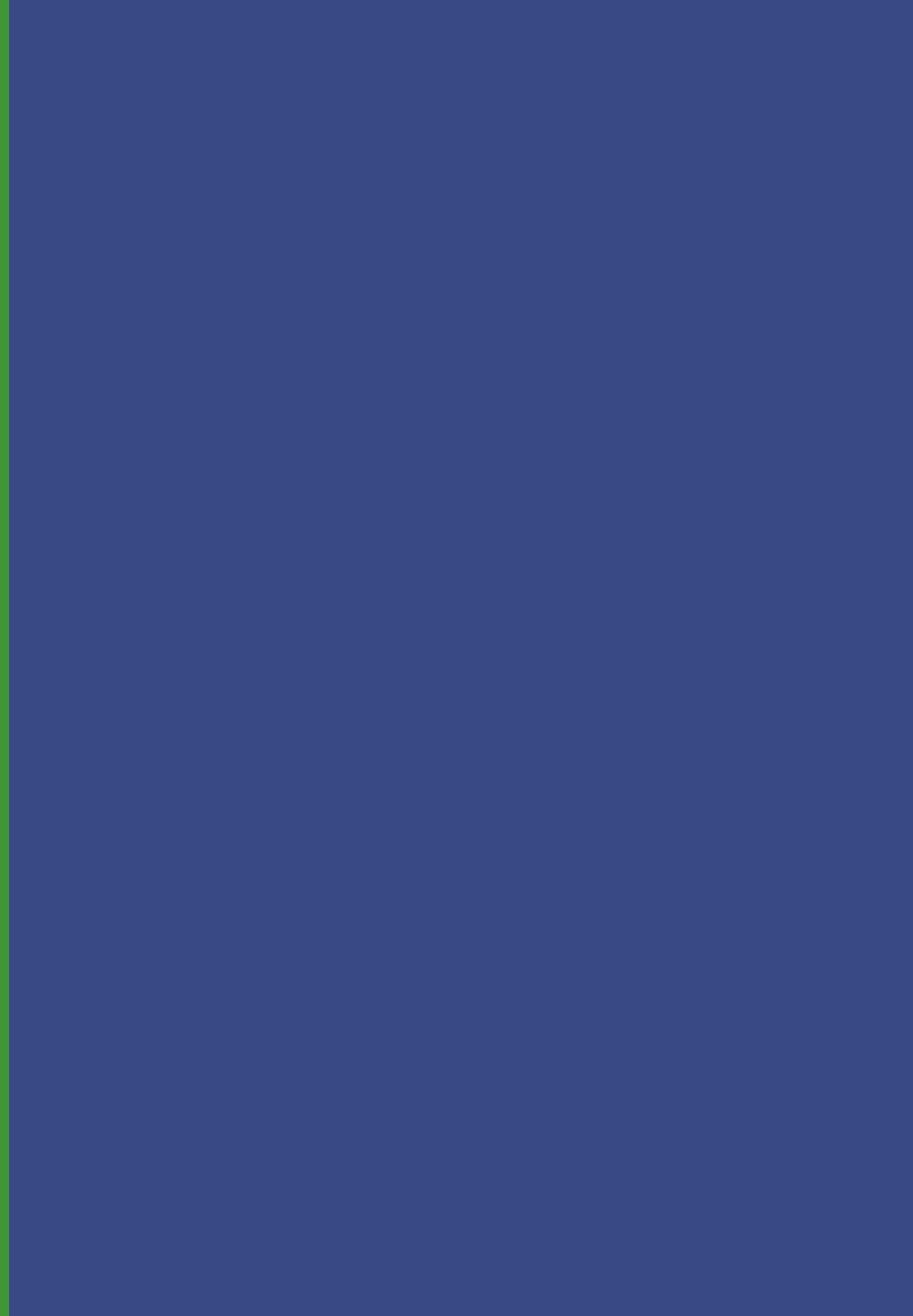

